University of Wollongong

Research Online

Faculty of Engineering and Information

Faculty of Engineering and Information

Sciences - Papers: Part A

Sciences

January 2015

\title{
Wall clutter mitigation using HOSVD in through-the-wall radar imaging with compressed sensing
}

Abdesselam Bouzerdoum

University of Wollongong, bouzer@uow.edu.au

Fok Hing Chi Tivive

University of Wollongong, tivive@uow.edu.au

Follow this and additional works at: https://ro.uow.edu.au/eispapers

Research Online is the open access institutional repository for the University of Wollongong. For further information contact the UOW Library: research-pubs@uow.edu.au 


\title{
Wall clutter mitigation using HOSVD in through-the-wall radar imaging with compressed sensing
}

\author{
Abstract \\ This paper addresses the problem of wall clutter mitigation in through-the-wall radar imaging using \\ compressed sensing. In the proposed method, the radar signals are recovered using a joint Bayesian \\ sparse representation, and the estimated coefficients are transformed into a third-order data tensor. Then, \\ higher-order singular value decomposition (HOSVD) is applied to form a multilinear wall subspace. To \\ remove the returns associated with wall clutter, the radar signal is projected onto the complement of the \\ wall subspace. Furthermore, a compact image formation model is developed using principal component \\ analysis (PCA), which yields a smaller dictionary size and reduced noise. Experimental results show that \\ the proposed HOSVD-based method outperforms the standard SVD-based wall clutter mitigation \\ technique.
}

\section{Keywords}

clutter, mitigation, hosvd, wall, radar, sensing, imaging, compressed

\section{Publication Details}

A. Bouzerdoum \& F. Hing Chi. Tivive, "Wall clutter mitigation using HOSVD in through-the-wall radar imaging with compressed sensing," in IEEE International Conference on Digital Signal Processing (DSP), 2015, pp. 85-89. 


\title{
Wall Clutter Mitigation using HOSVD in Through-the-Wall Radar Imaging with Compressed Sensing
}

\author{
Abdesselam Bouzerdoum and Fok Hing Chi Tivive \\ School of Electrical, Computer, and Telecommunications Engineering \\ University of Wollongong, Wollongong, NSW 2522, Australia \\ Email: \{a.bouzerdoum, tivive\}@uow.edu.au
}

\begin{abstract}
This paper addresses the problem of wall clutter mitigation in through-the-wall radar imaging using compressed sensing. In the proposed method, the radar signals are recovered using a joint Bayesian sparse representation, and the estimated coefficients are transformed into a third-order data tensor. Then, higher-order singular value decomposition (HOSVD) is applied to form a multilinear wall subspace. To remove the returns associated with wall clutter, the radar signal is projected onto the complement of the wall subspace. Furthermore, a compact image formation model is developed using principal component analysis (PCA), which yields a smaller dictionary size and reduced noise. Experimental results show that the proposed HOSVDbased method outperforms the standard SVD-based wall clutter mitigation technique.
\end{abstract}

\section{INTRODUCTION}

Over the past decade, there has been an increasing interest in through-the-wall radar imaging (TWRI) for its wide range of potential civilian and military applications, including searchand-rescue, law enforcement, and urban surveillance and reconnaissance [1]-[4]. A TWRI system can be used to detect behind-the-wall stationary targets and determine the building layout from images formed from the radar returns. A wide array aperture and a large signal bandwidth are required for high-resolution imaging, at the expense of prolonged data acquisition time and increased data storage. Thus, there is a demand for image formation methods that can reconstruct the scene with compressive measurements while maintaining high image quality.

Compressed sensing (CS) has shown that a signal or an image can be reconstructed at sub-Nyquist rate [5]. A number of techniques have been developed for efficient image reconstruction from reduced radar measurements [6]-[13]. Some of these CS-based methods assume that the measurements are free from the front wall returns, or the latter have been removed using background subtraction [6]-[9]. Others combine wall clutter mitigation and image formation [13]-[16]. In [13], CS is firstly applied to recover the missing radar measurements and then a wall clutter mitigation method, using spatial filtering [17] or a subspace projection technique [18], is applied to remove the wall returns. In [14] and [15], sparse representation is used to first estimate the signal coefficients, and then singular value decomposition (SVD) is applied directly on the estimated coefficients in order to segregate the wall returns from the target signal. In the SVD-based wall clutter mitigation method [14], [15], [18], the wall and target subspaces are obtained from a two-dimensional data matrix, where one dimension represents the signal (or coefficient) index and the other represents the antenna index. However, since the number of signal samples is often greater than the number of antennas, reducing the latter decreases the rank of the matrix, thus making it harder to separate the target subspace from the wall subspace. In [19], the discrete prolate spheroidal sequence (DPSS) was used to estimate the wall returns from reduced measurements. It was assumed that the strong wall reflections and the dominant wall reverberations last up to a distance of $1.5 \mathrm{~m}$ away from the wall; this distance is determined from EM simulations [20]. This range was used to estimate the DPSS coefficients associated with the wall returns. The problem is that the target reflections located within this range will also be removed or significantly attenuated.

In this paper, we propose to use HOSVD to represent the wall subspace. HOSVD was developed for processing multidimensional data arrays [21], [22]. Since its introduction in the mid-1990's, it has been successfully applied in many research areas, including independent component analysis [23], web link analysis [24], text representation [25], parameter estimation [26], and image denoising [27]. Here, we use it to separate the wall reflections from the target returns with a set of reduced measurements, i.e., reduced frequency samples and number of antennas. In the proposed method, a sparse representation is used for signal recovery. The recovered coefficients are arranged into a 3-way array, where the third dimension represents the basis index. Using HOSVD, a multilinear subspace is formed to characterize the wall reflections. Then, a subspace projection method is applied to remove the wall returns from the recovered signals. Finally, principal component analysis (PCA) is used to design a compact image formation model that can be easily solved with CS techniques.

The remainder of the paper is organized as follows. Section II introduces the TWRI signal model. Section III describes the proposed wall clutter mitigation technique using HOSVD. Section IV presents the experimental results, and Section V concludes the paper. 


\section{TWRI SignAL MODEL}

This section describes briefly the signal model of a throughthe-wall monostatic stepped-frequency radar. Consider an $N$-element linear synthetic aperture radar (SAR) for TWRI. The antenna transmits and receives a wideband steppedfrequency signal comprising $M$ frequencies, $\omega_{m}(m=$ $1, \ldots, M)$. Suppose that there are $P$ targets behind the wall. Assuming a point target model, the radar signal received by the $n$th antenna at the $m$ th frequency can be expressed as

$$
y_{n, m}=\sum_{k=1}^{L} \sigma_{n, \mathrm{w}} A_{k} e^{-j \omega_{m} \tau_{n, \mathrm{w}}^{k}}+\sum_{i=1}^{P} \sigma_{i} e^{-j \omega_{m} \tau_{n, i}}+\epsilon_{n, m},
$$

where $\sigma_{n, \mathrm{w}}$ is the complex reflectivity of the wall, $\tau_{n, \mathrm{w}}^{1}$ is the propagation delay associated with the direct return from the wall, $\tau_{n, \mathrm{w}}^{k}(k>1)$ is the propagation delay associated with the $k$ th wall reverberation, $A_{k}$ is the path loss factor of the $k$ th wall return, $L$ is the number of wall reverberations, $\sigma_{i}$ is the complex reflectivity of the $i$ th target, $\tau_{n, i}$ is the two-way propagation delay between the $n$th antenna and the $i$ th target, and $\epsilon_{n, m}$ is the measurement noise.

In CS-based methods for TWRI, a sensing matrix is used to obtain a subset of space-frequency measurements [6]-[11], [13]. Let $\Phi_{n} \in \mathbb{R}^{M_{1} \times M}\left(M_{1}<M\right)$ denote a sensing matrix in which each row has only one non-zero element indicating the selected frequency. The reduced measurements at the $n$th antenna location can be written in matrix-vector form as

$$
\boldsymbol{z}_{n}=\Phi_{n} \boldsymbol{y}_{n}
$$

where $\boldsymbol{y}_{n}=\left[y_{n, 1}, \ldots, y_{n, M}\right]^{T}$ and ${ }^{T}$ denotes the transpose operator. Applying an image formation method directly to the reduced measurements given in (2) generates an image with heavy wall clutter. Therefore, wall clutter mitigation is required before forming the radar image.

\section{Wall Clutter Mitigation using HOSVD}

In the proposed method, the radar signals are recovered using a joint Bayesian sparse representation. Then, HOSVD is applied to the tensor obtained from the estimated coefficients to form a multilinear wall subspace. Finally, the wall returns are mitigated by performing an orthogonal projection onto the wall subspace.

\section{A. Joint Signal Coefficient Estimation}

The received signal at each antenna, $\boldsymbol{y}_{n}$, can be sparsely represented using a dictionary $W=\left[\boldsymbol{w}_{1}, \ldots, \boldsymbol{w}_{R}\right]$ containing $R$ basis functions,

$$
\boldsymbol{y}_{n}=W \boldsymbol{x}_{n}+\boldsymbol{\epsilon}_{n}
$$

where $\boldsymbol{\epsilon}_{n}=\left[\epsilon_{n, 1}, \ldots, \epsilon_{n, M}\right]^{T}$ is the noise vector and $\boldsymbol{x}_{n}=$ $\left[x_{n, 1}, \ldots, x_{n, R}\right]^{T}$ is a sparse vector of signal coefficients. Using the sensing matrix $\Phi_{n}$, the reduced measurement vector can be expressed as

$$
\boldsymbol{z}_{n}=D_{n} \boldsymbol{x}_{n}+\widetilde{\boldsymbol{\epsilon}}_{n}
$$

where $D_{n}=\Phi_{n} W$ and $\widetilde{\boldsymbol{\epsilon}}_{n}=\Phi_{n} \boldsymbol{\epsilon}_{n}$.
The vector $\boldsymbol{x}_{n}$ can be recovered independently from each antenna measurements. However, in [16] it was shown that recovering radar signals jointly achieves better reconstruction accuracy than recovering each signal independently. Therefore, the joint Bayesian sparse framework is adopted here to estimate the signal coefficients $\boldsymbol{x}_{n}$.

Assuming that the noise term in (4) is zero-mean Gaussian with independent and identically distributed (i.i.d) components, the probability density function (pdf) of $\widetilde{\epsilon}_{n}$ is given by

$$
p\left(\widetilde{\boldsymbol{\epsilon}}_{n}\right)=\prod_{i=1}^{M_{1}} \mathcal{N}\left(\widetilde{\epsilon}_{n, i} \mid 0, \beta^{-1}\right),
$$

where $\beta$ is the noise precision. The likelihood of $\boldsymbol{x}_{n}$ is a multivariate Gaussian function,

$$
p\left(\boldsymbol{z}_{n} \mid \boldsymbol{x}_{n}, \beta\right)=(2 \pi / \beta)^{-M_{1} / 2} e^{-\frac{\beta}{2}\left\|\boldsymbol{z}_{n}-D_{n} \boldsymbol{x}_{n}\right\|^{2}} .
$$

To exploit the correlations among the signals, a shared prior is imposed on the coefficient vector $\boldsymbol{x}_{n}$,

$$
p\left(\boldsymbol{x}_{n} \mid \boldsymbol{\alpha}\right)=\prod_{i=1}^{R} \mathcal{N}\left(x_{n, i} \mid 0, \alpha_{i}^{-1}\right),
$$

where $\boldsymbol{\alpha}=\left[\alpha_{1}, \ldots, \alpha_{R}\right]$ is a vector of hyper-parameters. The posterior of $\boldsymbol{x}_{n}$ is a multivariate Student-t distribution with the mean and covariance given by [28]:

$$
\boldsymbol{\mu}_{n}=\Sigma_{n} D_{n}^{T} \boldsymbol{z}_{n}
$$

and

$$
\Sigma_{n}=\left(D_{n}^{T} D_{n}+A\right)^{-1},
$$

where $A=\operatorname{diag}\left(\alpha_{1}, \ldots, \alpha_{R}\right)$. The shared hyper-parameter vector $\widetilde{\boldsymbol{\alpha}}$ is estimated by maximizing the marginal likelihood [28], [29]. The estimate of the coefficient vector $\widetilde{\boldsymbol{x}}_{n}$ is given by the mean vector in (8):

$$
\widetilde{\boldsymbol{x}}_{n}=\left.\boldsymbol{\mu}_{n}\right|_{\boldsymbol{\alpha}=\widetilde{\boldsymbol{\alpha}}} .
$$

\section{B. Multilinear Wall Subspace}

The basis of the dictionary and the estimated coefficients are converted into a data tensor. At the $n$th antenna location, the basis functions weighted by their corresponding estimated coefficients are arranged into a matrix $Y_{n}$ :

$$
Y_{n}=\left[\boldsymbol{w}_{1} \widetilde{x}_{n, 1}, \ldots, \boldsymbol{w}_{R} \widetilde{x}_{n, R}\right], \quad n=1, \ldots, N .
$$

These matrices are then stacked one behind the other to form a mode-3 tensor, $\mathcal{Y}=\left\{Y_{1}, \ldots, Y_{N}\right\} \in \mathbb{C}^{M \times R \times N}$. Using HOSVD, the data tensor $\mathcal{Y}$ can be decomposed as

$$
\mathcal{Y}=\mathcal{C} \times{ }_{1} U^{(1)} \times{ }_{2} U^{(2)} \times{ }_{3} U^{(3)},
$$

where $\mathcal{C}$ is the core tensor, $\times_{i}, i=1,2,3$ is the mode- $i$ product, $U^{(i)}=\left[\boldsymbol{u}_{1}^{(i)}, \ldots, \boldsymbol{u}_{k_{i}}^{(i)}\right]$ is the unitary matrix of mode- $i$ matricization of tensor $\mathcal{Y}$, and $k_{i}$ is the number of singular vectors associated with mode- $i$. In [30] and [31], we have shown that the strong wall returns and the wall reverberations are captured by the first few dominant singular vectors, and some weak wall components are carried by the non-dominant 
singular vectors. The following steps are performed to identify the singular vectors spanning the wall subspace. First, a vector space $V^{i, j}$ is formed with the $j$ th singular vector of mode- $i$ :

$$
V^{i, j}=\boldsymbol{u}_{j}^{(i)}\left(\boldsymbol{u}_{j}^{(i)}\right)^{H},
$$

where ${ }^{H}$ denotes the Hermitian transpose. Then, the data tensor $\mathcal{Y}$ is projected onto the vector space $V^{i, j}$

$$
\widehat{\mathcal{Y}}^{i, j}=\mathcal{Y} \times_{i} V^{i, j} .
$$

From the matrix $\widehat{Y}_{n, i, j}$ extracted from the tensor $\widehat{\mathcal{Y}}^{i, j}$ we compute the radar signal $\widehat{\boldsymbol{y}}_{n, i, j}$ as follows:

$$
\widehat{y}_{n, i, j}(k)=\sum_{l=1}^{R} \widehat{Y}_{n, i, j}(k, l) .
$$

The radar signal $\widehat{\boldsymbol{y}}_{n, i, j}$ is converted into a range profile (RP) by applying an inverse fast Fourier transform. From the location of the main peak, the $j$ th singular vector of mode- $i$ can be classified into the wall and target classes. If the position of the main peak of the RP is between the radar location and the wall location, the singular vector is considered to span the wall subspace. More details on the estimation of the wall location and the classification of the singular vectors can be found in [31]. This classification technique is repeated for each singular vector to determine the multilinear wall subspace.

\section{Wall Clutter Mitigation by Subspace Projection}

A subspace projection is performed onto the complement of the wall subspace to remove the wall returns from the recovered signals. Let $\mathcal{W}_{i}$ be the index set of wall singular vectors associated with mode- $i$ and $\widehat{U}^{(i)}=\left\{\mathbf{u}_{j}^{(i)}\right\}_{j \in \mathcal{W}_{i}}$ be a matrix of singular vectors formed from the index set of $\mathcal{W}_{i}$. The subspace orthogonal to the wall subspace of mode- $i$ is computed as

$$
S_{\perp}^{(i)}=I-\widehat{U}^{(i)}\left(\widehat{U}^{(i)}\right)^{H},
$$

where $I$ denotes the identity matrix. For wall clutter mitigation, the data tensor $\mathcal{Y}$ is projected onto the multilinear orthogonal subspace

$$
\overline{\mathcal{Y}}=\mathcal{Y} \times{ }_{1} S_{\perp}^{(1)} \times_{2} S_{\perp}^{(2)} \times_{3} S_{\perp}^{(3)} .
$$

Finally, the radar signal $\overline{\boldsymbol{y}}_{n}$, free of wall clutter, are reconstructed from the tensor $\overline{\mathcal{Y}}$ using (15).

\section{Compact Image Formation Model}

In this section, we formulate a compact linear model that maps the recovered signals to the image of the scene. In [10] and [13], the sensing matrix $\Phi_{n}$ was used to reduce the measurements after the wall returns have been removed. Here, principal component analysis (PCA) is used to compress the recovered signals, reduce the dictionary size, and remove noise. Let $C$ denote the covariance matrix of $Z=\left[\overline{\boldsymbol{y}}_{1}, \ldots, \overline{\boldsymbol{y}}_{N}\right]$. Using the eigendecomposition, we can write $C=U \boldsymbol{\Lambda} U^{H}$, where $U=\left[\boldsymbol{u}_{1}, \ldots, \boldsymbol{u}_{M}\right]$ is a matrix of eigenvectors and $\boldsymbol{\Lambda}$ is a diagonal matrix of eigenvalues, arranged in descending order. We define a projection matrix
$\mathbf{P}=\left[\boldsymbol{u}_{1}, \ldots, \boldsymbol{u}_{K}\right]$ consisting of the first $K(K \ll M)$ eigenvectors. The Akaike Information Criterion (AIC) or Minimum Description Length (MDL) technique can be used to determine the $K$ eigenvectors that capture most of the target information [32]. The new compressed measurements associated with the $n$th antenna is given by $\overline{\boldsymbol{z}}_{n}=\mathbf{P}^{H} \overline{\boldsymbol{y}}_{n}$. To establish the link between the radar signals and the interrogated scene, the scene is partitioned into $Q$ pixels, and the image pixels are arranged into a vector $s \in \mathbb{C}^{Q \times 1}$. Let $\tau_{n, q}$ denote the focusing delay between the $n$th antenna and the $q$ th pixel. Assuming the target consists of points located precisely on the image pixels, the radar signal received by the $n$th antenna can be written as

$$
\bar{z}_{n}=\Omega_{n} s
$$

where $\Omega_{n}=\mathbf{P}^{H} \Psi_{n}$ and $\Psi_{n}=\left[\psi_{n}(m, q)\right]$ is an $M \times Q$ matrix with the $m q$ th element given by $\psi_{n}(m, q)=\exp \left(-j \omega_{m} \tau_{n, q}\right)$. Now, the relation between the measurements collected across the array aperture and the image of the scene $s$ can be expressed as

$$
\overline{\mathbf{z}}=\Omega s,
$$

where $\overline{\mathbf{z}}=\left[\overline{\boldsymbol{z}}_{1}^{T}, \ldots, \overline{\boldsymbol{z}}_{N}^{T}\right]^{T}$ and $\boldsymbol{\Omega}=\left[\Omega_{1}^{T}, \ldots, \Omega_{N}^{T}\right]^{T}$. To highlight the target from the clutter pixels, a filtering operation is incorporated into the model:

$$
\overline{\mathbf{z}}=\widetilde{\boldsymbol{\Omega}} \boldsymbol{\theta},
$$

where $\widetilde{\Omega}=\boldsymbol{\Omega} F, F$ is a sparse matrix containing the filter coefficients, and $\boldsymbol{\theta}$ is the estimated sparse vector. For image formation, we solve the following minimization problem:

$$
\min \|\boldsymbol{\theta}\|_{1} \quad \text { subject to }\|\overline{\mathbf{z}}-\widetilde{\boldsymbol{\Omega}} \boldsymbol{\theta}\|_{2} \leq \delta,
$$

where $\delta$ is a noise bound. After applying a CS recovery technique to solve (21), the formed image $s$ is computed as

$$
s=F \boldsymbol{\theta} .
$$

\section{EXPERIMENTAL RESULTS}

Numerical simulations are performed using XFDTD software to evaluate the performance of the proposed HOSVDbased wall clutter mitigation method. The simulated TWRI scene comprises a dihedral placed at $(0,1.4) \mathrm{m}$ behind a homogeneous wall of thickness $0.15 \mathrm{~m}$ and dielectric constant 7.76. A linear antenna array is placed at a standoff distance of $1.5 \mathrm{~m}$ in front of the wall. Since in practice it is difficult to have perfect alignment between the antenna array and the wall surface, the antenna array is deliberately tilted at an angle of $3^{\circ}$ with respect to the wall surface. The scene is interrogated with a 41-element array using a modulated Gaussian pulse with a center frequency of $2.5 \mathrm{GHz}$ as an excitation signal. The time domain response is then converted into a steppedfrequency signal with 201 frequencies covering the frequency band $[2,3] \mathrm{GHz}$. To quantify the quality of the formed images, the target-to-clutter ratio (TCR) is computed for each formed image

$$
\mathrm{TCR}=\frac{1}{N_{\mathrm{t}}} \sum_{q \in A_{\mathrm{t}}}|I(q)|^{2} / \frac{1}{N_{\mathrm{c}}} \sum_{q \in A_{\mathrm{c}}}|I(q)|^{2},
$$


where $|I(q)|^{2}$ is the squared magnitude of the $q$ th pixel, $A_{\mathrm{t}}$ is the target region, $A_{\mathrm{c}}$ is the clutter region, $N_{\mathrm{c}}$ and $N_{\mathrm{t}}$ are the number of pixels in the clutter and target regions, respectively. The clutter region is considered to be the entire image, excluding the target region.

In the first experiment, we compare the HOSVD with the SVD based method for wall clutter mitigation. Discrete prolate spheroidal sequence basis is used to generate the dictionary $W$ in (3) for signal recovery; the DPSS has been shown to be effective in approximating bandpass signals [33], [34]. For the sake of convenience, delay-and-sum (DS) beamforming is used to reconstruct the image in this experiment. A monte carlo simulation of 200 runs is performed to compute the TCR as a function of the number of reduced measurements. Figure 1 shows the TCR of the formed image as a function of the ratio of selected antennas, and Fig. 2 depicts the TCR of the formed image as a function of the ratio of selected frequencies. The results of both figures show that the HOSVD-based method outperforms the SVD-based method.

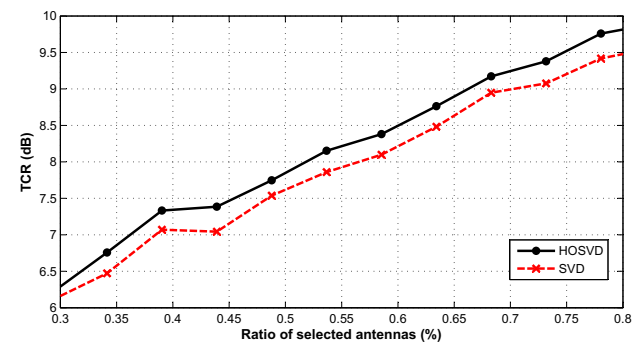

Fig. 1. TCR of the beamformed image as a function of the ratio of selected antennas $\left(N_{1} / N\right)$, where $N_{1}$ is the number of selected antennas. For each experiment, $30 \%$ of the total number of frequencies $(M=201)$ are selected.

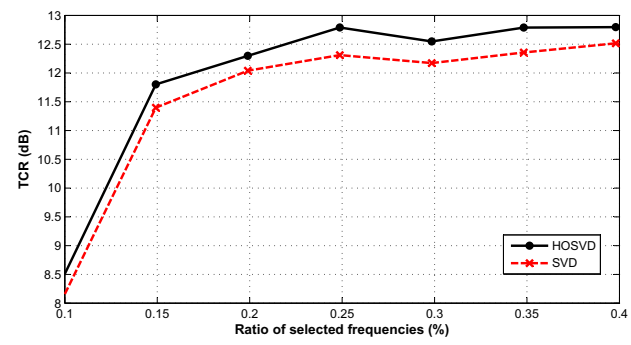

Fig. 2. TCR of the beamformed image as a function of the ratio of selected frequencies $\left(M_{1} / M\right)$. For each experiment, $36 \%$ of the total number of antennas $(N=41)$ are used to interrogate the scene.

Next, we use the Bayesian compressed sensing technique to solve (21) for reconstructing the image of the scene. A set of 1,800 measurements, i.e., 30 antennas and 60 frequencies are used to form an image of size $54 \times 91$ pixels. The proposed HOSVD-based method is used to mitigate the wall clutter. Here, the compact and standard models are used for image formation. In the former model PCA is applied to compress the measurement vector, whereas in the latter model the sensing matrix $\Phi_{n}$ is used to obtain the reduced measurements. The filtering operation is added to both image formation models, and the matrix $F$ is designed using a $3 \times 3$ Gaussian kernel. Table I lists the TCR and the dictionary size of the CS- based method using different image formation models. The TCR of the image and the size of the dictionary are obtained after averaging over 10 simulation runs. The compact image formation model achieves a smaller dictionary but higher TCR than the standard model. Figure 3 presents the images formed using DS beamforming and the CS-based method with different image formation models. The image obtained from the compact model has fewer false target pixels than the standard model.

TABLE I

TARGET TO CLUTTER RATIO OF THE CS-BASED METHOD USING DIFFERENT IMAGE FORMATION MODELS.

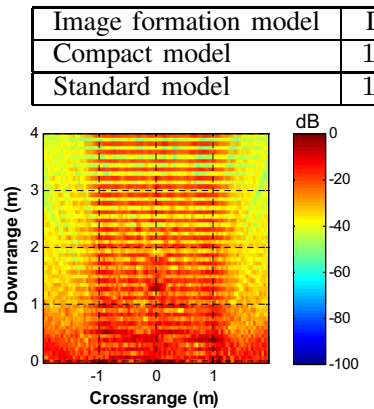

(a)

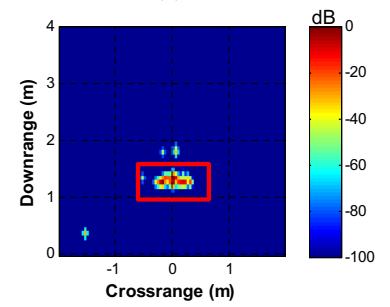

(c)

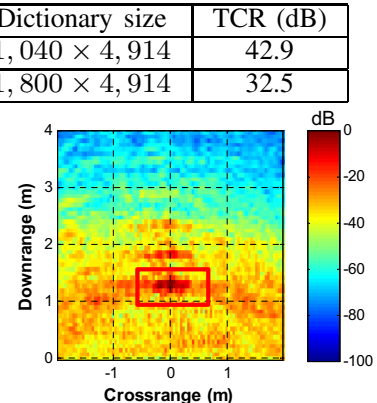

(b)

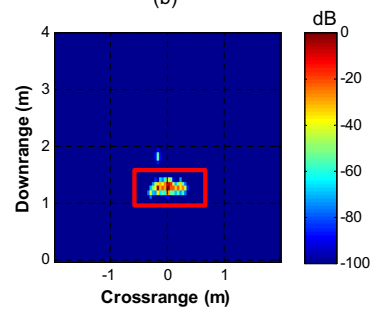

(d)
Fig. 3. Images of the TWRI scene reconstructed from $21.8 \%$ of the 8,241 measurements (41 antennas $\times 201$ frequencies): (a) and (b) DS beamformed images before and after wall clutter mitigation, (c) image formed using the standard image formation model, and (d) image obtained using the compact image formation model.

\section{CONCLUSION}

An improved subspace-based wall clutter mitigation method using HOSVD was developed for TWRI with compressed measurements. The proposed method applies a sparse representation to recover the radar signals where the estimated coefficients are converted into a data tensor. HOSVD is applied to form a multi-linear subspace capturing the wall contributions. Then, a subspace projection technique is employed to mitigate the wall returns. A compact linear model using PCA is designed for image formation. This model can reduce the dimension of the measurement vector and the size of the dictionary based on the target information contained in the recovered signals. Experimental results showed that the proposed HOSVD-based method can effectively remove the wall reflections and related reverberation from signals obtained with reduced measurements.

\section{ACKNOWLEDGMENT}

This work is supported by a grant from the Australian Research Council (ARC). 


\section{REFERENCES}

[1] M. G. Amin ( Ed.), Through-the-wall radar imaging. Boca Raton, FL, USA: CRC Press, 2010.

[2] F. Ahmad, M. G. Amin, and S. A. Kassam, "Synthetic aperture beamformer for imaging through a dielectric wall," IEEE Transactions on Aerospace and Electronic Systems, vol. 41, no. 1, pp. 271-283, 2005.

[3] F. Ahmad, Y. Zhang, and M. G. Amin, "Three-dimensional wideband beamforming for imaging through a single wall," IEEE Geoscience and Remote Sensing Letters, vol. 5, no. 2, pp. 176-179, 2008.

[4] M. G. Amin (Ed.), Compressive Sensing for Urban Radar. Boca Raton, FL, USA: CRC Press, 2014.

[5] E. J. Candes and M. B. Wakin, "An introduction to compressed sampling," IEEE Signal Processing Magazine, vol. 25, no. 2, pp. 21-30, 2008.

[6] Y.-S. Yoon and M. G. Amin, "Imaging of behind the wall targets using wideband beamforming with compressive sensing," Proc. IEEE/SP Workshop on Statistical Signal Processing, pp. 93-96, Aug. 31- Sep. 3, 2009.

[7] _ , "Through-the-wall radar imaging using compressive sensing along temporal frequency domain," Proc. IEEE International Conference on Acoustics Speech and Signal Processing (ICASSP), pp. 2806-2809, Mar. 14-19, 2010.

[8] Q. Huang, L. Qu, B. Wu, and G. Fang, "UWB through-wall imaging based on compressive sensing," IEEE Transactions on Geoscience and Remote Sensing, vol. 48, no. 3, pp. 1408-1415, 2010.

[9] M. Leigsnering, C. Debes, and A. M. Zoubir, "Compressive sensing in through-the-wall radar imaging," Proc. IEEE International Conference on Acoustics Speech and Signal Processing, pp. 4008-4011, May 22-27, 2011.

[10] M. G. Amin and F. Ahmad, "Compressive sensing for through-the-wall radar imaging," Journal of Electronic Imaging, vol. 22, no. 3, pp. 1-20, 2013.

[11] V. H. Tang, A. Bouzerdoum, and S. L. Phung, "Two-stage throughthe-wall radar image formation using compressive sensing," Journal of Electronic Imaging, vol. 22, no. 2, Article no. 021006, pp. 1-10, 2013.

[12] J. Qian, F. Ahmad, and M. G. Amin, "Joint localization of stationary and moving targets behind walls using sparse scene recovery," Journal of Electronic Imaging, vol. 22, no. 2, Article no. 021002, pp. 1-10, 2013.

[13] E. Lagunas, M. G. Amin, F. Ahmad, and M. Najar, "Joint wall mitigation and compressive sensing for indoor image reconstruction," IEEE Transactions on Geoscience and Remote Sensing, vol. 51, no. 2 , pp. 891-906, 2013.

[14] F. H. C. Tivive, A. Bouzerdoum, and V. H. Tang, "Multi-stage compressed sensing and wall clutter mitigation for through-the-wall radar image formation," Proc. IEEE 8th Sensor Array and Multichannel Signal Processing Workshop (SAM), pp. 489-492, Jun. 22-25, 2014.

[15] A. Bouzerdoum, F. H. C. Tivive, and V. H. Tang, "Multi-polarization through-the-wall radar imaging using joint bayesian compressed sensing," Proc. IEEE 19th International Conference on Digital Signal Processing (DSP), pp. 783-788, Aug. 20-23, 2014.

[16] V. H. Tang, A. Bouzerdoum, S. L. Phung, and F. H. C. Tivive, "Enhanced wall clutter mitigation for compressed through-the-wall radar imaging using joint bayesian sparse signal recovery," Proc. IEEE International Conference on Acoustics, Speech and Signal Processing (ICASSP), pp. 7804-7808, May 4-9, 2014.

[17] Y.-S. Yoon and M. G. Amin, "Spatial filtering for wall-clutter mitigation in through-the-wall radar imaging," IEEE Transactions on Geoscience and Remote Sensing, vol. 47, no. 9, pp. 3192-3208, 2009.

[18] F. H. C. Tivive, A. Bouzerdoum, and M. G. Amin, "An svd-based approach for mitigating wall reflections in through-the-wall radar imaging," Proc. IEEE Radar Conference, pp. 519-524, May 23-27, 2011.

[19] F. Ahmad, J. Qian, and M. G. Amin, "Wall clutter mitigation using discrete prolate spheroidal sequences for sparse reconstruction of indoor stationary scenes," IEEE Transactions on Geoscience and Remote Sensing, vol. 53, no. 3, pp. 1549-1557, 2014.

[20] N. S. F. Solicitation, "Radio frequency (RF) modeling of layered composite dielectric building materials," pp. 94-95. Available: http://www.acq.osd.mil/osbp/sbir/solicitations/sbir20081/navy081.pdf

[21] L. D. Lathauwer, B. D. Moor, and J. Vandewalle, "A multilinear singular value decomposition," SIAM Journal on Matrix Analysis and Applications, vol. 21, no. 4, pp. 1253-1278, 2000.
[22] G. Bergqvist and E. G. Larsson, "The higher-order singular value decomposition: theory and an application," IEEE Signal Processing Magazine, vol. 27, no. 3, pp. 151-154, 2010.

[23] L. D. Lathauwer, B. D. Moor, and J. Vandewalle, "Independent component analysis and (simultaneous) third-order tensor diagonalization," IEEE Transactions on Signal Procesing, vol. 49, no. 10, pp. 2262-2271, 2001.

[24] T. Kolda and B. Bader, "The TOPHITS model for higher-order web link analysis," Proc. SIAM Data Mining Conference Workshop on Link Analysis, Counterterrorism and Security, pp. 725-728, 2006.

[25] N. Liu, B. Zhang, J. Yan, Z. Chen, W. Liu, F. Bai, and L. Chien, "Text representation: from vector to tensor," Fifth IEEE International Conference on Data Mining, pp. 725-728, nov. 27-30, 2005.

[26] M. Haardt, F. Roemer, and G. D. Galdo, "Higher-order svd-based subspace estimation to improve the parameter estimation accuracy in multidimensional harmonic retrieval problems," IEEE Transactions on Signal Processing, vol. 56, no. 7, pp. 3198-3213, 2008.

[27] A. Rajwade, A. Rangarajan, and A. Banerjee, "Image denoising using the higher order singular value decomposition," IEEE Transactions on Pattern Analysis and Machine Intelligence, vol. 35, no. 4, pp. 849-862, 2012.

[28] S. Ji, D. Dunson, and L. Carin, "Multitask compressed sensing," IEEE Transactions on Signal Procesing, vol. 57, no. 1, pp. 92-106, 2009.

[29] Q. Wu, Y. D. Zhang, M. G. Amin, and B. Himed, "Complex multitask bayesian compressed sensing," IEEE International Conference on Acoustics, Speech, and Signal Processing, pp. 3375-3379, May 4-9, 2014.

[30] F. H. C. Tivive and A. Bouzerdoum, "An improved svd-based wall clutter mitigation method for through-the-wall radar imaging," Proc. IEEE 14th Workshop on Signal Processing Advances in Wireless Communications (SPAWC), pp. 430-434, Jun. 16-19, 2013.

[31] F. H. C. Tivive, A. Bouzerdoum, and M. G. Amin, "A subspace projection approach for wall clutter mitigation in through-the-wall radar imaging," IEEE Transactions on Geoscience and Remote Sensing, vol. 53, no. 4, pp. 2108-2122, 2015.

[32] M. Wax and T. Kailath, "Detection of signals by information theoretic criteria," IEEE Transactions on Acoustics Speech Signal Processing, vol. 33, no. 2, pp. 387-392, 1985.

[33] D. Slepian, "Prolate spheroidal wave functions, fourier analysis, and uncertainty - V: the discrete case," The Bell System Technical Journal, vol. 57, no. 5, pp. 1371-1430, 1978.

[34] M. A. Daveport and M. B. Wakin, "Compressive sensing of analog signals using discrete prolate spheroidal sequences," Applied and Computational Harmonic Analysis, vol. 33, no. 3, pp. 438-472, 2012. 